\section{Enzyme therapy}

THE lung secretions of cystic-fibrosis sufferers with respiratory bacterial infections contain large amounts of neutrophil-derived DNA; such secretions are consequently much more viscous than normal. S. Shak et al. (Proc. natn. Acad. Sci. U.S.A. 87, 9188-9192; 1990) have now cloned and expressed human recombinant DNase I, and show that it can dramatically reduce the viscosity of the thickened sputum in vitro but has no effect on the mucus of unaffected individuals. Taken in aerosol form, the pure human DNase I may prove to be a safe and effective way of aiding the daily clearance of sputum in patients suffering from cystic fibrosis. Preliminary clinical studies are said to be encouraging.

\section{Bang on}

WE may be living in an unstable Universe that could explode because of a chance collision between cosmic rays, J. Ellis, $A$. Linde and $M$. Sher suggest. They make this rather extraordinary claim in a paper in Physics Letters (B252, 203-211; 1990 ) in which they try to pin down the mass of two of the most important asyet-undiscovered fundamental particles, the top quark and the Higgs boson. Although the authors come to no conclusions about the masses of these particles, they do put forward ideas about the consequences of certain plausible values. In particular, if the top quark's mass is greater than some critical value determined by the mass of the Higgs boson, the Universe is not stable but rather is trapped in a long-lived unstable state out of which it could be precipitated by some violent event. Cosmic rays could provide the necessary trigger, and whether they have failed yet to do so because the Universe is insufficiently unstab!e, or because of sheer chance, is the big question.

\section{Blooming genes}

MOLECULAR genetic analysis of flower development continues apace with the report from E.S. Coen et al. (Cel/63, $1311-1322 ; 1990)$ of the cloning of a snapdragon gene that seems to be a master switch in the initial specification of flower-forming meristems. In plants with mutations in the gene (known as floricaula, or flo for short), vegetative meristems still give rise to inflorescence meristems, but these fail to produce flower-forming meristems and instead generate additional indeterminate shoots. Examination of the sequence of the flo protein product has not yet revealed any notable similarities to known protein sequences, though near the $\mathrm{N}$ terminus is a region strikingly rich in proline residues. The new work follows closely on the cloning of two 'flower homeotic' genes, which specify organs within a flower - at some level, presumably, genes of this class must come under flo's control.

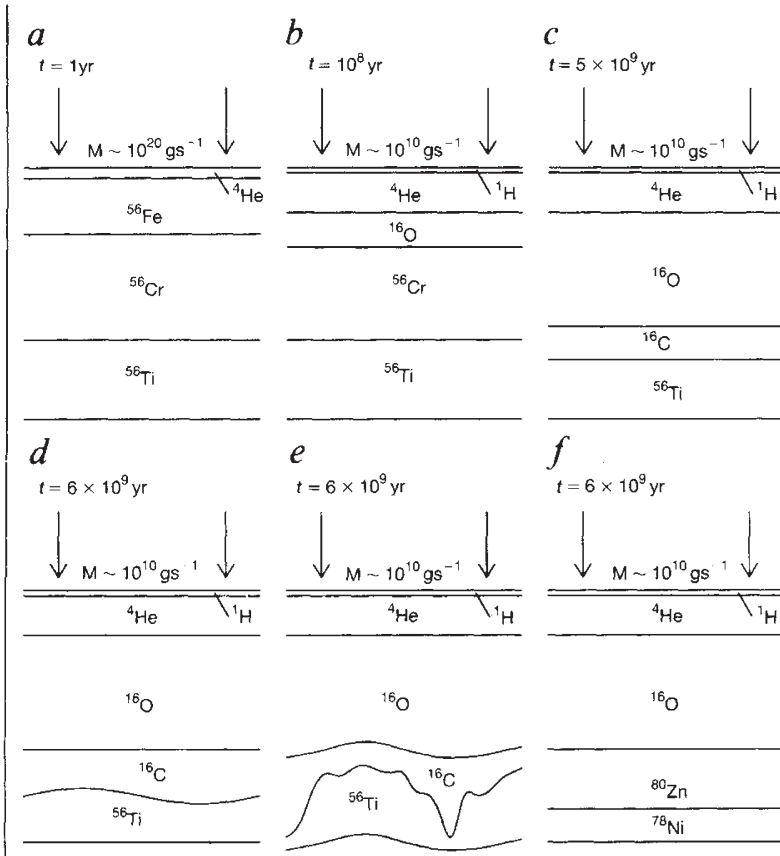

FIG. 2 Schematic sketch of one possible history of the surface layers of an isolated neutron star (from Blaes et al. ${ }^{1}$ ). Following its formation in a supernova event at time $t=0$ and a brief subsequent episode of rapid accretion from the surrounding supernova remnant, the star settles down to a much lower rate of accretion from the interstellar medium. The chemical composition of the surface layers is determined by the rates of various pycnonuclear (density-induced fusion) reactions as the accreted material is gradually compressed to ever higher densities. An appreciable density inversion eventually forms at the interface between carbon and titanium (note that in these layers the nuclei form a body-centred-cubic lattice in a sea of relativistic degenerate electrons). The interface becomes unstable at $t=6 \times 10^{9} \mathrm{yr}$, resulting in overturn and the release of gravitational and nuclear energy. This energy propagates to the stellar surface as seismic waves and may then lead to the emission of $\gamma$-radiation from the magnetosphere (see text).

demonstrate that once an inversion becomes sufficiently strong, the interface becomes unstable to an clastic RaylcighTaylor mode (Fig. 2). Finally, they argue that only a small fraction (about $10^{-3}$ ) of the neutron star's surface 'breaks' in any given event, resulting in a localized overturn of the accreted matter and the release of $10^{35}-10^{+1}$ ergs of gravitational and (mostly) nuclcar energy.

This is just about the right amount of energy per event if $\gamma$-ray bursts originate on neutron stars in our own Galaxy. Moreover, variations in the size and shape of the quaking region, together with variations in the effects of projection and neutron-star rotation, may account for many of the complexities and case-to-case differences in the profiles of $\gamma$-ray bursts (in sharp contrast with the phenomenon of cosmic X-ray bursts, whose burst profiles are generally much smoother and more regular). Most importantly, the continual accretion provides a replenishable source of fuel for driving at least $10^{\circ}$ bursts during the lifetime of every isolated neutron star in the Galaxy, as required for consistency with the frequency with which bursts are observed.
The energy release occurs so deeply beneath the neutron star's surface that very little of the energy is propagated to the photosphere via clectron conduction or other thermal processes, nor do the surface layers expand appreciably. In a preceding paper ${ }^{8}$, some of the authors argued that the energy is conveyed to the surface mainly by seismic processes. There, the disturbed neutron star's magnetic field couples the released energy to the magnetosphere by generating intense, relativistic Alfvén waves. The final piece of the picture, namely, the conversion of these waves into relativistic particle motions and thence to $\gamma$-rays, constitutes an immensely difficult problem and has not yet been studied with any degree of rigour. Nevertheless, Blaes et al. ${ }^{\circ}$ were able to present various plausibility arguments that the available energy can be efficiently converted to $\gamma$ radiation on a timescale consistent with $\gamma$-ray burst phenomenology.

If the basic picture painted by Blaes et al. ${ }^{1.8}$ turns out to be correct, we may be witnessing the emergence of a unified theoretical framework for understanding the phenomena of $\gamma$-ray burst sources, X-ray burst sources, X-ray pulsars and radio pulsars. According to this view, $\gamma$-ray bursters are mostly isolated neutron stars that are accreting matter very slowly from the interstellar medium. Most X-ray bursters are neutron stars that are accreting matter at higher rates (perhaps $10^{16} \mathrm{~g} \mathrm{~s}^{-1}$ ) from low-mass binary companion stars and that burn their nuclear fuel in discrete flashes at relatively shallow depths (tens of metres) beneath the neutron-star photosphere. The transfer of the thermonuclear energy released in a flash to the neutron-star photosphere via thermal processes leads to the emission of an X-ray burst.

1. Blaes, O. Blandford, R Madau, P \& Koonin S. Astrophys, J. 363, 612-627 (1990)

2. Harwit, M. \& Salpeter. E. E. Astrophys, J. 186, L37 (1973)

3. Hameury, J. M., Bonazzola, S., Heyvaerts, J. \& Ventura, J. Astr. Astrophys. 111, 242-251 (1982).

Woosley, S. E. \& Wallace, R. K. Astrophys. J. 258 $716-732$ (1982).

5. Pacini, F. \& Ruderman, M. Nature 251, 399-400 (1974).

6. Tsygan, A. I. Astr. Astrophys. 44, 21-24 (1975)

7. Fabian, A. C., Icke, V. \& Pringle, J. E. Astrophys. Space Sci. 42, $77-81$ (1976)

8. Blaes, O., Blandford, R., Goldreich, P. \& Madau, P. Astro phys. J. 343, 839-848 (1989)

9. Mazets, E. P., Golenetskii, S. V., Il'inskii, V. N., Aptekar, R. L. \& Guryan, Yu. A. Nature 282, 587 - 589 (1979) 